\title{
Quantifying reconnective activity in braided vector fields
}

\author{
Christopher Prior and Anthony R. Yeates* \\ Department of Mathematical Sciences, Durham University, \\ Lower Mountjoy, Stockton Road, Durham DH1 3LE, United Kingdom
}

(Dated: July 17, 2018)

\begin{abstract}
We introduce a novel technique for evaluating the changing connectivity of a vector field whose integral curves (field lines) form tangled tubular bundles. Applications of such fields include magnetic flux ropes, relativistic plasma jets, stirred two-dimensional fluids, super-fluid vortices, and polymer networks. The technique is based on maps of the field line winding - the average entanglement of a given field line with all other field lines. Previously this had been developed for divergence-free vector fields. By extending some previous theoretical results, we show how it can be applied to any vector field that forms a tubular bundle. We demonstrate the efficacy of this technique on data from laboratory plasma experiments with two interacting magnetic flux ropes. Performed in the UCLA Large Plasma Device, the plasma's magnetic field structure is too complex to identify a single dominant current sheet as an expected site of magnetic reconnection. Previously, this complex structure had restricted the ability to analyze the evolving magnetic connectivity, but this is no such restriction to our method. We demonstrate that the plasma establishes a periodically oscillating cycle of magnetic field structure variation which, whilst triggered by an ideal instability, is dominated by magnetic reconnection. This reconnection leads to periodically varying coherence of a merged central flux rope, a conclusion supported by analysis of the writhing structure of the magnetic field.
\end{abstract}

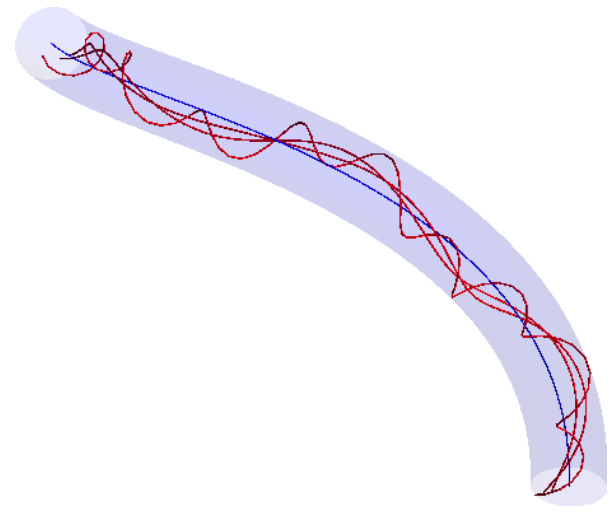

FIG. 1: A braided vector field whose entangled field lines are contained within a tubular domain.

\section{INTRODUCTION}

Many physical systems can be represented as braided vector fields, where the term braiding here refers to entanglement of the field's integral curves (field lines). These vector fields are contained within a tubular volume as in Figure 1. Braided fields arise in plasma physics as magnetic flux ropes, in two-dimensional fluids as entangled particle trajectories (e.g. [1]), knotted, canopy or superfluid vorticies [2-7], and in physical networks such as elastic filaments [8-10].

In the plasma context, understanding the changing connectivity (reconnection) of the magnetic field is fundamental to understanding many astrophysical phenomena. An important example is the coronal heating problem in

\footnotetext{
* christopher.prior@durham.ac.uk
}

the Sun's atmosphere (corona), where energy released by reconnection is believed to account for exceptionally high temperatures [11-16]. Further afield, magnetic reconnection is the supposed source of gamma ray bursts in relativistic jets, a critical observational tool for probing their internal magnetic structure (e.g. [17]). There is also significant interest in probing the fundamental nature of magnetic reconnection in laboratory plasmas (e.g. [18-20]).

It is, therefore, often critical to quantify the changing connectivity of a braided vector field. With a finite number of curves (e.g. fluid stirrer trajectories) this can be done using mathematical braid invariants $[1,21]$. However, for space filling vector fields there is no real meaning to the notion of individual field line reconnection - only a reconnection density - and we cannot directly apply methods associated with discrete braided curves.

Topological quantities such as the magnetic helicity and kinetic helicity are invariant if the fluid in question satisfies the Euler equations [22], under which the field lines are deformed by isotopy and cannot reconnect. Thus significant changes in these quantities indicate reconnective activity. Unfortunately they are scalar quantities integrated over the whole vector field; they measure the average entanglement of the field. Even if the average value is conserved, a field can experience significant reconnection. This is the case for the evolution of coronal magnetic flux ropes, where studies show that magnetic fields can spontaneously re-organize themselves through reconnection so as to minimize their energy [15, 23-31].

Progress in this area has been aided by the development of a quantity we call here the field line winding, the average entanglement of a single field line with the rest of the field [32-34]. Its distribution amongst all field lines of a braided field is a complete topological invariant, meaning changes in connectivity occur if and only if this 
distribution changes. In a study of relaxation through magnetic reconnection, Russell et al. [30] demonstrated that the evolution of field line winding follows an advective law to leading order, giving novel insight as to how highly entangled magnetic fields in plasmas can spontaneously self-organize to minimize their energy. However, field line winding has previously only been defined for divergence-free fields [32-34]. We show here that it can be used to evaluate reconnective activity in any differentiable braided vector field. Thus, similar analysis could be applied to a much wider variety of physical systems.

In the magnetic field context, the field line winding is not the only approach which has been used to measure changing connectivity. Hesse and co-authors [35, 36] showed that reconnecting flux (field line reconnection weighted by magnetic flux) can be measured by the integrated parallel electric field through localized sites. This is the so called GMR (General Magnetic Reconnection) theory. Contemporary use of GMR often involves identifying sheets of significant current (Quasi separatrix layers/QSL's) at which reconnection will occur in magnetized plasmas (e.g. [20, 37-39]). More direct approaches for have been applied by Wilmot-Smith and De Moortel [40] and Mackay and van Ballegooijen [41], who track the changing connectivity between particular field line "types" (specific to each numerical experiment). We highlight the fact that in both approaches the field has to be separated into distinct subsections (either current sheets, or discrete "types") before connectivity can be defined and tracked. The approach we develop here does not require either assumption to analyze reconnection.

We demonstrate the utility of the field line winding by applying it to a pair of evolving magnetic flux ropes induced in a laboratory plasma in the UCLA Large Plasma Device (LaPD). The group have conducted a number of such experiments [18, 19, 42, 43]. DeHaas and Gekelman [39] were able to analyze helicity flows in and out of a dominant QSL established between the interacting ropes. In another study Gekelman [20] used the GMR theory to measure reconnective activity.

Here we focus on the "two-moon" experiment in which the flux ropes are placed significantly closer together (Figure 2 of [44]). The flux ropes coalesce to form a magnetic field with complex small-scale current structure, i.e. there is no clear single QSL at which reconnection occurs. We will demonstrate that the field line winding measure can be used to analyze the changing connectivity even in the absence of a clearly dominant reconnection site.

\section{MEASURING THE CHANGING CONNECTIVITY OF VECTOR FIELDS}

In this section we set out our general approach for measuring changes in field line connectivity in braided vector fields. Braided fields are defined here as vector fields $\mathbf{B}$, on a domain foliated by a family of flat planes $\left\{\mathcal{D}_{z} \mid z \in[0, h]\right\}$. Braided fields $\mathbf{B}$ are tangent on the side

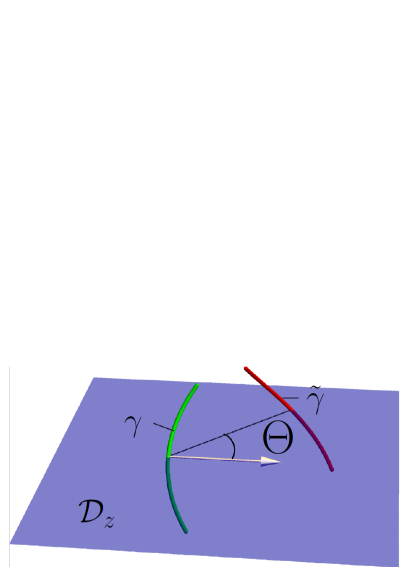

(a)

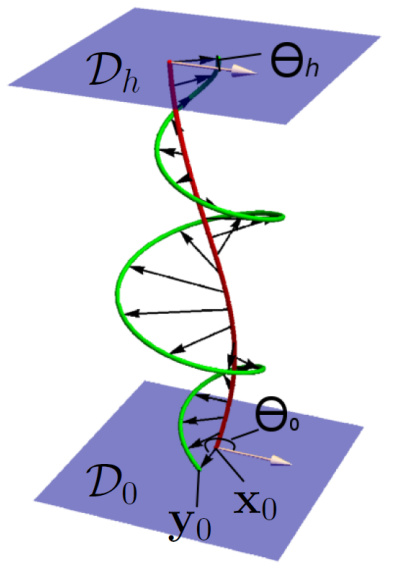

(b)
FIG. 2: Geometrical interpretation of $\mathcal{L}\left(\mathbf{x}_{0}, t\right)$, showing the definition of the angle $\Theta$ between the curves $\gamma$ and

$\widetilde{\gamma}$ in each plane, relative to the $x_{1}$ direction (white arrows).

boundaries $\left\{\partial \mathcal{D}_{z} \mid z \in[0, h]\right\}$ but have some non-zero positive distribution $\mathbf{B} \cdot \hat{\mathbf{z}}>0$ on every plane $\mathcal{D}_{z}$, including the end boundaries. This ensures that the field lines $\gamma\left(\mathbf{x}_{0}, s\right)$, obtained by solving the ODE

$$
\frac{\mathrm{d} \gamma}{\mathrm{d} s}=\frac{\mathbf{B}\left(\gamma\left(\mathbf{x}_{0}, s\right)\right)}{\left|\mathbf{B}\left(\gamma\left(\mathbf{x}_{0}, s\right)\right)\right|}
$$

with initial condition $\gamma\left(\mathbf{x}_{0}, 0\right)=\mathbf{x}_{0} \in \mathcal{D}_{0}$, must flow through the domain (i.e. pierce each plane $\mathcal{D}_{z}$ only once). We can define a coordinate system for this domain $\left(x_{1}, x_{2}, z\right)$ where $\left(x_{1}, x_{2}\right)$ span the planes $\mathcal{D}_{z}$ and $z$ labels the particular plane $\mathcal{D}_{z}$. We can also parametrize any field line by its start point $\mathbf{x}_{0} \in \mathcal{D}_{0}$ and $z$ coordinate.

\section{A. The field line winding $\mathcal{L}$}

To measure a braided field's changing connectivity, we define the field line winding $\mathcal{L}\left(\mathbf{x}_{0}, t\right)$, for a field line $\gamma\left(\mathbf{x}_{0}, z\right)$ with respect to all other field lines $\widetilde{\gamma}\left(\mathbf{y}_{0}, z\right)-$ rooted at all other end-points $\mathbf{y}_{0} \in \mathcal{D}_{0}$ - as the quantity

$$
\mathcal{L}\left(\mathbf{x}_{0}, t\right)=\int_{\gamma} \frac{\mathrm{d} \mathcal{L}\left(\mathbf{x}_{0}, t\right)}{\mathrm{d} z} \mathrm{~d} z
$$

where

$$
\begin{aligned}
& \frac{\mathrm{d} \mathcal{L}\left(\mathbf{x}_{0}, t\right)}{\mathrm{d} z}=\frac{1}{2 \pi} \int_{\mathcal{D}_{0}} \frac{\mathrm{d} \Theta(\widetilde{\gamma}, \gamma, z)}{\mathrm{d} z} \mathrm{~d}^{2} y_{0}, \\
& \Theta(\widetilde{\gamma}, \gamma, z)=\arctan \left(\frac{\widetilde{\gamma}_{2}\left(\mathbf{y}_{0}, z\right)-\gamma_{2}\left(\mathbf{x}_{0}, z\right)}{\widetilde{\gamma}_{1}\left(\mathbf{y}_{0}, z\right)-\gamma_{1}\left(\mathbf{x}_{0}, z\right)}\right) .
\end{aligned}
$$

The angle $\Theta$ is measured in the plane $\mathcal{D}_{z}$, between the $x_{1}$-axis and a line joining $\gamma(z)$ to $\widetilde{\gamma}(z)$ (Figure 2a). Thus $\mathrm{d} \Theta(\widetilde{\gamma}, \gamma, z) / \mathrm{d} z$ measures the local rate at which the field 


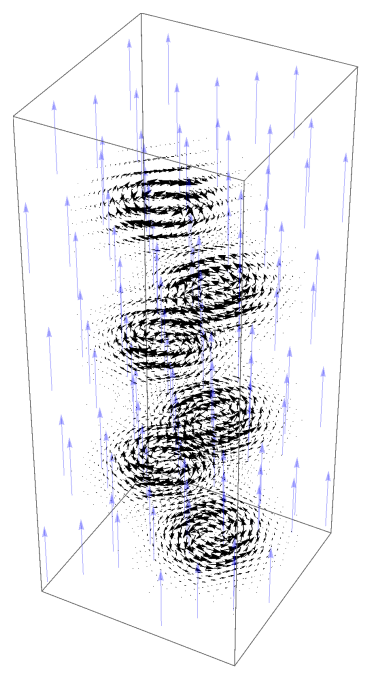

(a)

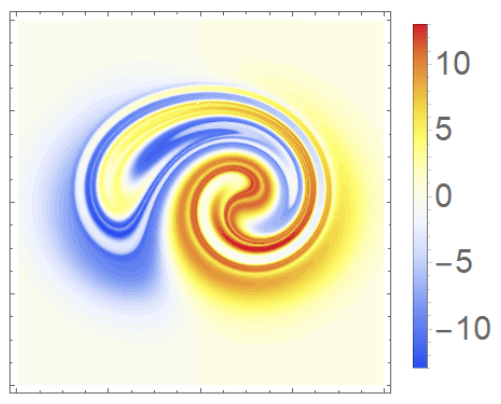

(b)

FIG. 3: A braided field used in [15]. (a) The field is a superposition of counter-rotating twisted field (black) and a straight background field (blue). (b) The distribution $\mathcal{L}\left(\mathbf{x}_{0}, t\right)$ for this field.

line passing through $\left(\widetilde{\gamma}_{1}\left(\mathbf{y}_{0}, z\right), \widetilde{\gamma}_{2}\left(\mathbf{y}_{0}, z\right), z\right)$ is winding round the field line $\gamma$. The integral in (3) is over $\mathcal{D}_{0}$, meaning that each point $\left(\widetilde{\gamma}_{1}, \widetilde{\gamma}_{2}, z\right)$ is a function of its initial point $\mathbf{y}_{0} \in \mathcal{D}_{0}$, thus to perform this calculation we must follow all field lines in the domain. If we imagine a limiting scenario with only two field lines $\gamma$ and $\Gamma$, then $\mathcal{L}\left(\mathbf{x}_{0}, t\right)$ reduces to

$$
\mathcal{L}\left(\mathbf{x}_{0}, t\right)=\frac{\Theta(\Gamma, \gamma, h)-\Theta(\Gamma, \gamma, 0)}{2 \pi}+n(\Gamma, \gamma)
$$

where $n(\Gamma, \gamma)$ is the signed integer number of times the curve $\Gamma$ winds around $\gamma$ (with right handed rotations positive and left handed negative). So $\mathcal{L}\left(\mathbf{x}_{0}, t\right)$ represents the number of times $\Gamma$ winds around $\gamma$ (in Figure $2 \mathrm{~b}$ we have $n(\Gamma, \gamma)=-2)$. For a space-filling vector field $\mathbf{B}, \mathcal{L}\left(\mathbf{x}_{0}, t\right)$ represents the average winding of all other field lines of B with the field line $\gamma$. By computing $\mathcal{L}\left(\mathbf{x}_{0}, t\right)$ on the whole of $\mathcal{D}_{0}$ we define the mutual winding connectivity of all field lines of $\mathbf{B}$.

The simplest possible distribution $\mathcal{L}\left(\mathbf{x}_{0}, t\right)$ is a uniformly-twisted field, which would have $\mathcal{L}\left(\mathbf{x}_{0}, t\right)=$ constant. A more interesting example is the braid-based vector field studied in $[15,30]$, whose structure consists of a series of counter twists (Figure 3a). It has a mean $\mathcal{L}\left(\mathbf{x}_{0}, t\right)$ value of 0 , but a significantly complex distribution of $\mathcal{L}\left(\mathbf{x}_{0}, t\right)$ including winding of both signs, as shown in Figure 3(b). The experimental magnetic field data in Section III will have aspects of both distributions.

\section{B. Measuring reconnection}

In Appendix A we demonstrate the following theorem.

Theorem II.1 Consider the braided vector fields $\mathbf{B}(\mathbf{x}, t)$ at times $t-\Delta t$ and $t$. The field lines of $\mathbf{B}(\mathbf{x}, t-\Delta t)$ and $\mathbf{B}(\mathbf{x}, t)$ can be linked by an isotopy which vanishes on the bounding planes $\mathcal{D}_{0}$ and $\mathcal{D}_{h}$ if and only if $\mathcal{L}\left(\mathbf{x}_{0}, t-\Delta t\right) \equiv$ $\mathcal{L}\left(\mathbf{x}_{0}, t\right), \forall \mathbf{x}_{0} \in \mathcal{D}_{0}$.

The main point is that a change in $\mathcal{L}\left(\mathbf{x}_{0}, t\right)$, for any $\mathbf{x}_{0}$, necessarily implies a change in the field line connectivity of $\mathbf{B}(\mathbf{x}, t)$.

The requirement that the isotopy vanish on $\mathcal{D}_{0}$ and $\mathcal{D}_{h}$ means that $\mathbf{B}(\mathbf{x}, t-\Delta t)$ must be reachable from $\mathbf{B}(\mathbf{x}, t)$ without moving the end-points of the field lines on these boundaries. In many applications, there is an underlying motion of the medium that would violate this requirement, yet would not change the connectivity of $\mathbf{B}(\mathbf{x}, t)$ because the field lines would be material lines under this motion. This is true for magnetic field lines in a plasma, or vortex lines in a fluid. It is desirable to remove this underlying ideal motion of the end-points, so as to measure only changes in connectivity occurring with respect to this ideal motion.

To do this, denote the underlying ideal flows on $\mathcal{D}_{0}$ and $\mathcal{D}_{h}$ by $\mathbf{V}^{0}\left(\mathbf{x}_{0}, t\right)$ and $\mathbf{V}^{h}(\mathbf{x}, t)$. To ensure that we are following the same bundle of field lines in time, we change the shape of $\mathcal{D}_{0}(t)$ and $\mathcal{D}_{h}(t)$ by evolving their boundaries under the flows of $\mathbf{V}^{0}$ and $\mathbf{V}^{h}$. Accordingly, the time derivative of $\mathcal{L}\left(\mathbf{x}_{0}, t\right)$ can be written as

$$
\begin{aligned}
& \frac{\mathrm{d} \mathcal{L}\left(\mathbf{x}_{0}, t\right)}{\mathrm{d} t}=\frac{\mathrm{d}}{\mathrm{d} t} \int_{0}^{h} \frac{1}{2 \pi} \int_{\mathcal{D}_{0}(t)} \frac{\mathrm{d} \Theta(\widetilde{\gamma}, \gamma, z)}{\mathrm{d} z} \mathrm{~d}^{2} y_{0} \mathrm{~d} z \\
& =\int_{0}^{h} \frac{1}{2 \pi} \int_{\mathcal{D}_{0}(t)} \frac{\partial}{\partial t}\left(\frac{\mathrm{d} \Theta(\widetilde{\gamma}, \gamma, z)}{\mathrm{d} z}\right) \mathrm{d}^{2} y_{0} \mathrm{~d} z+ \\
& \int_{0}^{h} \frac{1}{2 \pi} \oint_{\partial \mathcal{D}_{0}(t)} \frac{\mathrm{d} \Theta(\widetilde{\gamma}, \gamma, z)}{\mathrm{d} z} \mathbf{V}^{0}\left(\mathbf{y}_{0}, t\right) \cdot \hat{\mathbf{n}} \mathrm{d} y_{0} \mathrm{~d} z .
\end{aligned}
$$

The last term accounts for changing shape of $\partial \mathcal{D}_{0}(t)$, whereas the previous term accounts both for changes in winding due to reconnection but also changes due to ideal motions of the field line start/end points around one another. This term may be rewritten explicitly in terms of the net winding angles on the upper and lower boundaries 
as

$$
\begin{aligned}
& \int_{0}^{h} \frac{1}{2 \pi} \int_{\mathcal{D}_{0}(t)} \frac{\partial}{\partial t}\left(\frac{\mathrm{d} \Theta(\widetilde{\gamma}, \gamma, z)}{\mathrm{d} z}\right) \mathrm{d}^{2} y_{0} \mathrm{~d} z= \\
& \frac{1}{2 \pi} \int_{\mathcal{D}_{0}(t)}\left[\frac{\partial \Theta(\widetilde{\gamma}, \gamma, h)}{\partial t}-\frac{\partial \Theta(\widetilde{\gamma}, \gamma, 0)}{\partial t}\right] \mathrm{d}^{2} y_{0}+ \\
& \int_{\mathcal{D}_{0}(t)} \frac{\partial n(\widetilde{\gamma}, \gamma)}{\partial t} \mathrm{~d}^{2} y_{0},
\end{aligned}
$$

where $n(\tilde{\gamma}, \gamma)$ is the integer number of full windings between the field line curves $\widetilde{\gamma}$ and $\gamma$. If the evolution is isotopic, the corresponding integral vanishes as $n$ cannot change [45].

To remove the contribution from ideal motions, consider the distribution of $\mathcal{L}\left(\mathbf{x}_{0}, t\right)$ that would be obtained purely due to the ideal motion from a given distribution at time $t-\Delta t$. To do this, we track the same field line $\gamma$ by taking its start point $\mathbf{x}_{0}(\tau)$ for $\tau \in[t-\Delta t, t]$ to be the preimage of $\mathbf{x}_{0}$ under the flow of $\mathbf{V}^{0}$. Then the $\mathcal{L}\left(\mathbf{x}_{0}, t\right)$ distribution expected purely from the ideal motion would be

$$
\begin{aligned}
& \mathcal{L}^{\mathrm{id}}\left(\mathbf{x}_{0}, t\right)=\mathcal{L}\left(\mathbf{x}_{0}(t-\Delta t), t-\Delta t\right)+ \\
& \frac{1}{2 \pi} \int_{t-\Delta t}^{t}\left\{\int_{\mathcal{D}_{0}(\tau)}\left[\left.\frac{\partial \Theta(\widetilde{\gamma}, \gamma, h)}{\partial \tau}\right|_{\mathrm{id}}-\left.\frac{\partial \Theta(\widetilde{\gamma}, \gamma, 0)}{\partial \tau}\right|_{\mathrm{id}}\right] \mathrm{d}^{2} y_{0}\right. \\
& \left.+\oint_{\partial \mathcal{D}_{0}(\tau)} \frac{\mathrm{d} \Theta(\widetilde{\gamma}, \gamma, z)}{\mathrm{d} z} \mathbf{V}^{0}\left(\mathbf{y}_{0}, t\right) \cdot \hat{\mathbf{n}} \mathrm{d} y_{0}\right\} \mathrm{d} \tau
\end{aligned}
$$

where

$$
\begin{aligned}
& \left.\frac{\partial \Theta(\widetilde{\gamma}, \gamma, 0)}{\partial \tau}\right|_{\mathrm{id}}=\frac{\left[\mathbf{y}_{0}-\mathbf{x}_{0}(\tau)\right] \times \mathbf{V}^{0}\left(\mathbf{x}_{0}(\tau), \tau\right)}{\left|\mathbf{y}_{0}-\mathbf{x}_{0}(\tau)\right|^{2}}, \\
& \left.\frac{\partial \Theta(\widetilde{\gamma}, \gamma, h)}{\partial \tau}\right|_{\mathrm{id}}= \\
& \frac{\left[\widetilde{\gamma}\left(\mathbf{y}_{0}, h\right)-\gamma\left(\mathbf{x}_{0}(\tau), h\right)\right] \times \mathbf{V}^{h}\left(\gamma\left(\mathbf{x}_{0}(\tau), h\right), \tau\right)}{\left|\widetilde{\gamma}\left(\mathbf{y}_{0}, h\right)-\gamma\left(\mathbf{x}_{0}(\tau), h\right)\right|^{2}} .
\end{aligned}
$$

We can subtract this contribution from the observed $\mathcal{L}\left(\mathbf{x}_{0}, t\right)$ to measure the contribution from reconnective activity with respect to the underlying ideal motion, which we define as

$$
\mathcal{R}\left(\mathbf{x}_{0}, t\right)=\frac{\mathcal{L}\left(\mathbf{x}_{0}, t\right)-\mathcal{L}^{\mathrm{id}}\left(\mathbf{x}_{0}, t\right)}{\Delta t} .
$$

Non-zero $\mathcal{R}\left(\mathbf{x}_{0}, t\right)$ necessarily indicates a change of field line connectivity within the domain.

As a practical remark Theorem II.1, when applied to a finite domain, assumes that $\mathbf{B} \cdot \hat{\mathbf{n}}=0$ on the side boundary $\partial \mathcal{D}_{z}$. If field lines are allowed to enter or leave this side boundary, then this can change the apparent $\mathcal{L}\left(\mathbf{x}_{0}, t\right)$ measured within the domain. This is an additional complication that needs to be considered in applications; in the example presented in Section III, we find this not to be a significant effect, provided $\Delta t$ is small enough. However, it does place additional constraints on the domain $\mathcal{D}_{0}$, which must be chosen so that all field lines starting in this domain end within $\mathcal{D}_{h}$.

\section{APPLICATION TO RECONNECTING MAGNETIC FLUX TUBES}

As an example application of the reconnection measure $\mathcal{R}\left(\mathbf{x}_{0}, t\right)$, we apply it to study the interaction of magnetic flux ropes in a laboratory plasma experiment. The experiment was performed in the Large Plasma Device at UCLA [46], an $18 \mathrm{~m}$ long tube filled with plasma (helium in this case; shown in Figure 4a). The bulk of the plasma carries no net current. The flux ropes are created by a masked cathode source in the shape of two half discs (hence "two-moon"), separated by only $1 \mathrm{~cm}$ (by comparison the cross-section of the data we receive is $24 \mathrm{~cm} \times 24 \mathrm{~cm})$. The value of the currents was set sufficiently high that the flux ropes would become kinkunstable once established [44, 47]. More specific details of the experimental setup for collecting the data are given in [44]. Here we outline only the aspects critical or specific to our analysis.

\section{A. Experimental data format}

Three-axis magnetic and Mach (flow) probes were used to obtain time series of $\partial \mathbf{B} / \partial t$ (with estimated uncertainty $\pm 2 \%$ ) and $\mathbf{M}=\mathbf{V} / c_{s}$ (with estimated uncertainty $\pm 5 \%$ ), where $\mathbf{V}$ is the plasma velocity and $c_{s}$ is the ion sound speed. The experiment was repeated and the probes repositioned so as to build up three-dimensional arrays of $\partial \mathbf{B} / \partial t$ and $\mathbf{M}$, as functions of time.

From the $\partial \mathbf{B} / \partial t$ data the magnetic field $\mathbf{B}$ was extracted using the known initial background field $\mathbf{B}_{b}=$ $B_{0} \hat{\mathbf{z}}$, where $B_{0} \approx 300 \mathrm{G}$. To extract the plasma velocity $\mathbf{V}$, it is necessary to know the ion sound speed

$$
c_{s}=9.79 \times 10^{5} \sqrt{\frac{\gamma Z T_{e}}{\mu}} \mathrm{cm} \mathrm{s}^{-1},
$$

where we assume $\gamma Z=1$ and $\mu=4.003$ for helium. The electron temperature was measured as $T_{e}=4 \mathrm{eV}$ in the background plasma, and about $8 \mathrm{eV}$ in the center of the flux ropes. We approximate $T_{e}$ elsewhere by interpolating between these two values, proportional to the longitudinal current density $\left|J_{z}\right|$.

The data were measured on a grid with coordinates $\left(x_{1}, x_{2}, z\right)$ where $x_{1} \in[-12,11.76], x_{2} \in[-11.76,12]$, $z \in[64,1024]$ (all in $\mathrm{cm}$ ), with grid lengths $n_{1}=100$, $n_{2}=100, n_{3}=16$ respectively. The low resolution along the $z$ axis results from constraints on the measurement probe locations, but is acceptable because of the strong background "guide" field compared to the transverse field generated by the two-moon currents, typically of order $1 \mathrm{G}$. In this study we have data for a total duration $1.28 \mathrm{~ms}$, with 2000 equally spaced snapshots separated by $\Delta t=0.64 \mu \mathrm{s}$.

Critically, the data result from a series of repeat experiments which are averaged, owing to the need to repeat the experiment each time the probes are repositioned. 
Significant steps are taken to correlate the data spatially and temporally: see [44] for details. In general, the experiments are found to be repeatable, though there are some indications of chaos locally in the the system [44]. As such the averaged magnetic field has a small amount of divergence. The reconnective measure $\mathcal{R}\left(\mathbf{x}_{0}, t\right)$ does not require a divergence-free field, so we choose not to apply a correction to remove the divergence.

\section{B. Basic properties of the magnetic field}

1. The dominance of the background magnetic field strength over the current-generated component means $\mathbf{B} \cdot \hat{\mathbf{z}}>0$ everywhere in the domain. Thus the field lines will only travel along the tube in one direction (a requirement for braided fields).

2. The velocity field $\mathbf{V}$ does not vanish on the boundary of the domain (see Figure $4 \mathrm{~b}$ and c). Thus we will use (11) to evaulate the reconnective activity in the field.

3. The magnetic field forms a heavily tangled substructure at the centre of the domain, as the flux ropes rapidly merge - this structure is indicated in a "color map" in Figure 4(d). Briefly the field lines are colored by one of four sectors of $\mathcal{D}_{0}$ in which they begin. The color is then plotted where the field line ends on $\mathcal{D}_{h}$. The color mixing indicates a complex magnetic field topology; it appears to be folded rather than uniformly twisted (which would lead to a perfect spiral shape).

4. The current density (calculated by $\mathbf{J}=\nabla \times \mathbf{B}$ ) has significant sub-structure. This is shown in Figures 4(e) and (f), which are plots of local field line twisting due to the current, $(\nabla \times \mathbf{B}) \cdot \mathbf{B} /|\mathbf{B}|^{2}$, integrated along magnetic field lines for each start point $\mathbf{x}_{0} \in \mathcal{D}_{0}$. The majority of the twisting is positive, corresponding to the current injected to create the flux ropes, and localized in the center of the domain. There is significant small-scale variation which we would not expect for a uniformly twisted flux rope.

5. The current structure shows significant time variation. At $t=0.64 \mathrm{~ms}$ (Figure $4 \mathrm{e}$ ) there is a clear spiral structure with a central core of zero (net) current, while at $t=1.28 \mathrm{~ms}$ (Figure $4 \mathrm{f}$ ) the current is more clustered (though with significant substructure). In the latter half of the evolution, we found the current structure to alternate periodically between these two states.

6. We estimate the Alfvén speed as $v_{\mathrm{A}}=$ $B / \sqrt{\mu_{0} n_{i} m_{i}} \approx 2.5 \times 10^{5} \mathrm{~m} \mathrm{~s}^{-1}$, based on a number density $n_{i}=2 \times 10^{18} \mathrm{~m}^{-3}$. To estimate the Alfvén time we note field line are of the order $10 \mathrm{~m}$ length, so the Alfvén time is approximately $4 \times 10^{-5} \mathrm{~s}$. By contrast, the time between snapshots is $6.4 \times 10^{-7} \mathrm{~s}$, so the time discretization suffices to resolve ideal motions and hence discriminate these from reconnective motion.

\section{Quantities computed}

We calculate a set of quantities for the magnetic field at a cadence of 20 time steps, $\Delta t=0.0128 \mathrm{~ms}$. For each quantity, say $\mathcal{Q}, \mathcal{Q}\left(\mathbf{x}_{0}, t\right)$ will denote its distribution on $\mathbf{x}_{0} \in \mathcal{D}_{0}$ at a time $t$ (all quantities are integrated over field lines), and $\mathcal{Q}(t)=\frac{1}{\left|\mathcal{D}_{0}\right|} \int_{\mathcal{D}_{0}} \mathcal{Q}\left(\mathbf{x}_{0}, t\right) \mathrm{d}^{2} x_{0}$ its integrated value over the whole field, normalised by the area $\left|\mathcal{D}_{0}\right|$ at that time. This area changes due to ideal motions (as discussed in Section II B), but it is also slightly modified at each time so as to include only those field lines that reach the far $z$ boundary within the measured domain. For our small $\Delta t$, the change in $\left|\mathcal{D}_{0}\right|$ is generally less than $1 \%$, so that the effect on $\mathcal{L}\left(\mathbf{x}_{0}, t\right)$, for example, is insignificant. Further, we shall denote the unsigned integral of each quantity by $\mathcal{Q}_{\text {abs }}(t)=\frac{1}{\left|\mathcal{D}_{0}\right|} \int_{\mathcal{D}_{0}}\left|\mathcal{Q}\left(\mathbf{x}_{0}, t\right)\right| \mathrm{d}^{2} x_{0}$. The calculated quantities are:

1. Field line winding (weighted by the domain size). A value of $\mathcal{L}\left(\mathbf{x}_{0}, t\right)$ in the highly twisted central region corresponds to an average winding of about 0.147 turns.

2. Reconnective and ideal rates of change. We compute $\mathcal{R}\left(\mathbf{x}_{0}, t\right)$, from (11), using an edge-detection algorithm to track the changing shape of $\mathcal{D}_{0}$. In doing so we also record the rate of change in field line winding due to purely ideal end-point motions, which we label $\mathcal{I}\left(\mathbf{x}_{0}, t\right)$, i.e.

$\mathcal{I}\left(\mathbf{x}_{0}, t\right)=\frac{\mathcal{L}^{\mathrm{id}}\left(\mathbf{x}_{0}, t\right)-\mathcal{L}\left(\mathbf{x}_{0}(t-\Delta t), t-\Delta t\right)}{\Delta t}$.

3. Twist. The (average) local rotation of the field about a field line $\gamma$, as opposed to the winding of the whole field given by $\mathcal{L}$. It is

$$
\mathcal{T}\left(\mathbf{x}_{0}, t\right)=\int_{\gamma\left(\mathbf{x}_{0}\right)} \frac{\mathbf{J} \cdot \mathbf{B}}{\mathbf{B}^{2}} \mathrm{~d} s,
$$

where $s$ is arclength along $\gamma$ (see e.g. [45]). Positive values indicate right-handed rotation. It is this quantity (averaged over the length of the field line) which is shown in Figures 4(e) and (f).

4. Writhe. The writhe of a curve is a dimensionless measure of its contortion and is frequently used in astrophysical, biophysical and mechanical contexts. For braided fields it is given [45] by

$$
\mathcal{W}\left(\mathbf{x}_{0}, t\right)=\int_{\gamma\left(\mathbf{x}_{0}\right)} \frac{\hat{\mathbf{z}} \cdot \mathbf{T} \times \frac{\mathrm{d} \mathbf{T}}{\mathrm{d} z}}{1+|\hat{\mathbf{z}} \cdot \mathbf{T}|} \mathrm{d} z
$$




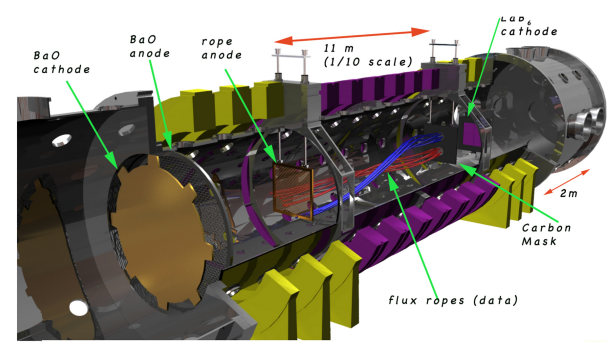

(a)

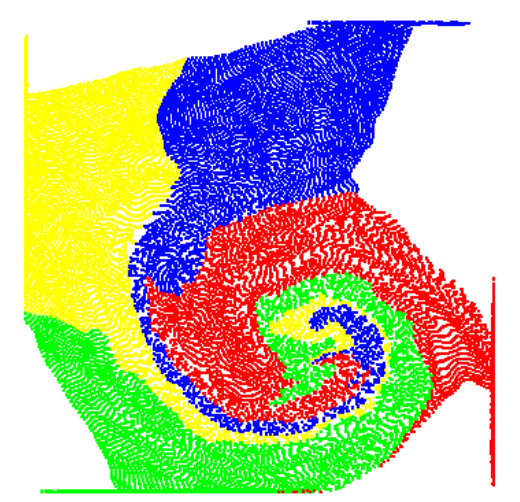

(d)

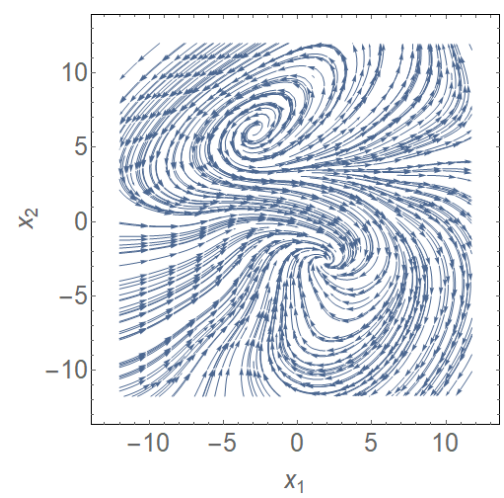

(b)

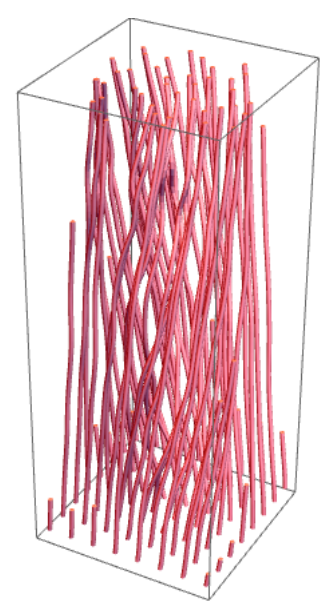

(c)

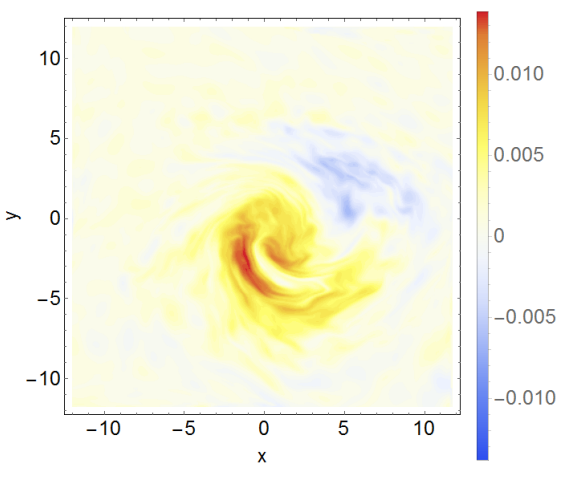

(e)

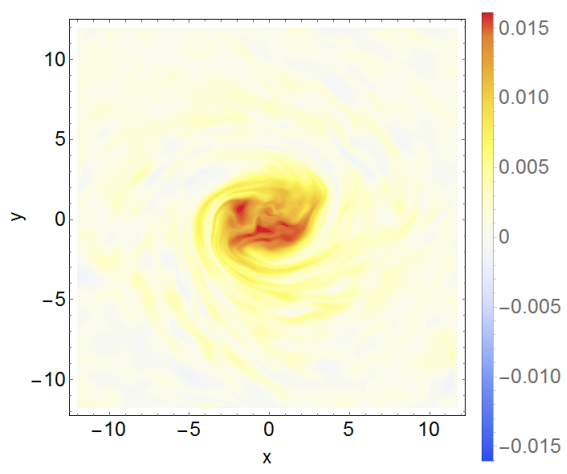

(f)

FIG. 4: The flux rope experiment, showing (a) the experimental setup (reproduced from [48]), (b) streamlines of the transverse velocity field $\mathbf{V}$ in the plane $\mathcal{D}_{h}$ at $t=0.64 \mathrm{~ms}$, (c) a small subset of the field lines of $\mathbf{B}$ at $t=1.28 \mathrm{~ms},(\mathrm{~d})$ a color map of the magnetic connectivity from $\mathcal{D}_{0}$ to $\mathcal{D}_{1}$ at $t=0.64 \mathrm{~ms}$, (e) the field-line integrated vertical current density at $t=0.64 \mathrm{~ms}$, and (f) the same at $t=1.28 \mathrm{~ms}$.

and is positive/negative for right/left handed helices. Because of the significant aspect ratio of our domain, the numerical values of $\mathcal{W}$ are small $(\approx 0.0004)$, but this does not mean that the geometrical effect is small. This is shown by the appropriately scaled curves in Figure 5.

Since we have a complex entangled field rather than a set of discrete flux tubes, we do not have the simple relationship $\mathcal{L}\left(\mathbf{x}_{0}\right)=\mathcal{W}\left(\mathbf{x}_{0}\right)+\mathcal{T}\left(\mathbf{x}_{0}\right)$ that holds for thin flux ropes [45, 49], but we do expect a certain amount of ideal twist-writhe conversion due to ideal instabilities of the interacting flux ropes.

\section{Results: integrated quantities and dominating reconnection}

We discuss first the time evolution of the integrated quantities; their spatial distribution will be discussed in Section III F.
Figure 6 shows many of the integrated quantities, as functions of time. In Figure 6(a) we see that $\mathcal{L}(t)$ generally increases, consistent with the continual input of positive twisting by the current injection as seen in Figures 4(e) and (f). There is initially uniform growth followed by regular periodic oscillations about a mean growth. Figure 6(b) shows the net rate of change due to reconnective activity, $\mathcal{R}(t)$. This is mainly negative, indicating that reconnection is (on average) acting to oppose the growth of positive winding due to the current input. After $t=0.64 \mathrm{~ms}$ the oscillations in $\mathcal{R}(t)$ have a similar amplitude to its average value, indicating significant variation in the nature of the reconnective contribution. Figure 6(c) shows the total absolute reconnective activity, $\mathcal{R}_{\text {abs }}(t)$, which peaks in the middle of the evolution. This always has an oscillatory component but the oscillation is more coherent after $t=0.8 \mathrm{~ms}$. As shown in Figure $6(\mathrm{~d})$, the net writhe $\mathcal{W}(t)$ also settles into a relatively regular cycle after $t=0.8 \mathrm{~ms}$.

Figure 6(e) compares the total unsigned contributions 


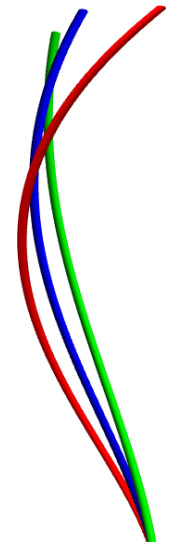

(a)

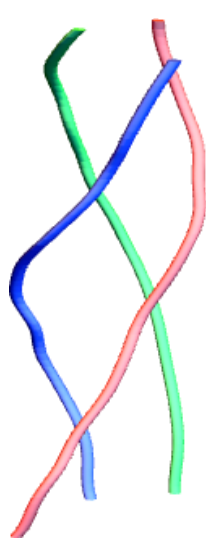

(b)

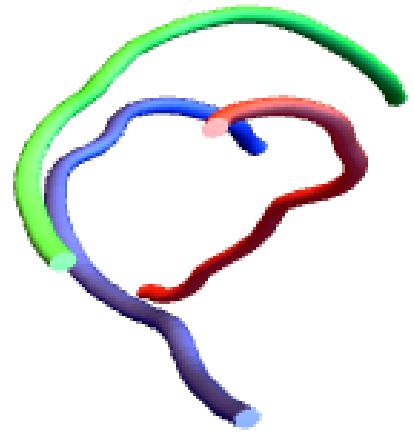

(c)
FIG. 5: Writhe values for field lines typical of the experimental data. (a) Helices with $\mathcal{W}=0.0004$ (red), 0.0002 (blue), and 0.0001 (green). (b) and (c) show field lines taken from the actual data (for $t=0.7936 \mathrm{~ms}$ ), with the same $\mathcal{W}$ values as in (a). Curves are plotted with the aspect ratios $(1,1,2.5)$ used in later figures.

from reconnection, $\mathcal{R}_{\text {abs }}(t)$, and from ideal end-point motions, $\mathcal{I}_{\text {abs }}(t)$. We see that changes in average winding due to reconnection are significantly larger than the ideal contribution. In Figure 6(f) we see that the difference in net contribution is smaller, although $\mathcal{R}_{\text {abs }}(t)$ still tends to dominate at later times. Overall, the changing connectivity is dominated by reconnective activity rather than by ideal loss due to fluid motion at the end boundaries.

\section{E. Results: twist-writhe conversion driving the evolution}

By re-scaling both $\mathcal{W}(t)$ and twist $T(t)$ between 0 and 1 (Figure 7a), we see they oscillate out of phase with minima of one coinciding with maxima of the other. This is reminiscent of the ideal twist-writhe conversion associated with the ideal kink instability of MHD/plasma physics, though since this is a non-ideal evolution their sum is not conserved.

This periodic oscillation has the same period (roughly $0.2 \mathrm{~ms}$ ) as the oscillations seen in $\mathcal{R}(t)$ (Figure $7 \mathrm{~b}$ ) and in $\mathcal{L}(t)$ (Figure 7c). Further, it appears that the local extrema in $\mathcal{W}(t)$ and $\mathcal{T}(t)$ occur just prior to those in the other quantities. This series of temporal coincidences is consistent with the idea that an ideal kink mechanism [47] is driving the oscillations in the later part of the evolution. For a thin plasma column with free boundaries and an axial plasma flow, Ryutov et al. [47] show that the frequency of the most unstable kink mode is

$$
k=\frac{|\mathbf{V}|}{2 L} \sqrt{1-\left(\frac{|\mathbf{V}|}{v_{\mathrm{A}}}\right)^{2}},
$$

where $L=11 \mathrm{~m}$ is the length of the column and $v_{\mathrm{A}}$ is the Alfvén speed. From the mean kinetic energy over the experiment, we estimate the average velocity to be $|\mathbf{V}| \approx$ $1.25 \times 10^{5} \mathrm{~cm} \mathrm{~s}^{-1}$. The Alfvén velocity was estimated earlier to be $v_{\mathrm{A}}=2.5 \times 10^{5} \mathrm{~m} \mathrm{~s}^{-1}$, leading to $k \approx 4.9 \mathrm{kHz}$. This is in good agreement with the observed oscillation, whose period $0.2 \mathrm{~ms}$ corresponds to a frequency of $5 \mathrm{kHz}$.

The fact that reconnective activity gives the dominant contribution to the change in field connectivity suggest that, while the ideal kink instability might trigger the oscillatory behaviour in the system, the complex topology of the merged fields means that this sets off a cycle of reconnective activity. This cycle must somehow force the magnetic field to rearrange its topology in the central region. We next explore this spatial evolution.

\section{F. Results: spatial distribution of topological quantities}

We focus on the phase $t>0.75 \mathrm{~ms}$ when periodic behaviour is prevalent in all global diagnostics. The qualitative behavior of the distributions $\mathcal{L}\left(\mathbf{x}_{0}, t\right)$, $\mathcal{R}\left(\mathbf{x}_{0}, t\right)$ and $\mathcal{W}\left(\mathbf{x}_{0}, t\right)$ repeats from one cycle to the next, so it suffices to illustrate one cycle. Snapshots of these three distributions at a sequence of times $0.7936 \mathrm{~ms}, 0.8448 \mathrm{~ms}, 0.896 \mathrm{~ms}, 0.9344 \mathrm{~ms}, 0.97280 \mathrm{~ms}$, covering one (local) minimum-to-minimum cycle of $\mathcal{L}(t)$, are shown in Figure 8 (for clarity $\mathcal{L}\left(\mathbf{x}_{0}, t\right)^{2}$ is plotted). The subset of high $\mathcal{L}$ values $\left(\mathcal{L}^{2}>0.0625\right)$ initially form a spiral shape (a). This "folds" inwards to form a more uniform central distribution ( $d$ and $g$ ), which is then "unravelled" to reform the spiral shape ( $\mathrm{j}$ and $\mathrm{m})$. At all times, a diffuse background of weak negative and positive reconnective activity is present outside of the more active central region, likely a result of interaction between the weakly twisted background and the core "flux rope".

In Figure 8, the quantity $\mathcal{R}\left(\mathbf{x}_{0}, t\right)$ is plotted as $\pm \sqrt{\left|\mathcal{R}\left(\mathbf{x}_{0}, t\right)\right|}$ so as to emphasize its features. Strong positive values $(\sqrt{|\mathcal{R}|}>90)$ are most prevalent when the spiral structure is forming, ( $b$ and $n$ ) and least prevalent when the $\mathcal{L}$ structure is most coherent (h). Further the high $\mathcal{R}$ densities occur at the centre of the spiral (c.f. (c)-(b)) where $\mathcal{L}\left(\mathbf{x}_{0}, t\right)$ is lowered. A more detailed analysis indicates that positive $\mathcal{R}$ creates the spiral arm while negative activity creates the lower- $\mathcal{L}$ gap inside the spiral. Thus reconnective activity is shaping the the field's topology.

The writhe distributions in the right-hand column of Figure 8 indicate that the majority of significantly contorted curves lie in the central flux rope region. In (c) and (o) we see the same (but weak) spiral structure observed in the $\mathcal{L}$ plots, whilst when the $\mathcal{L}$ structure is most coherent we see a similarly compact structure for the $\mathcal{W}$ distribution (i). Intriguingly, there is always a region in the middle of this high- $\mathcal{W}$ region with almost no $\mathcal{W}$, even for (i) where the link distribution $\mathcal{L}$ is close to uniform. 


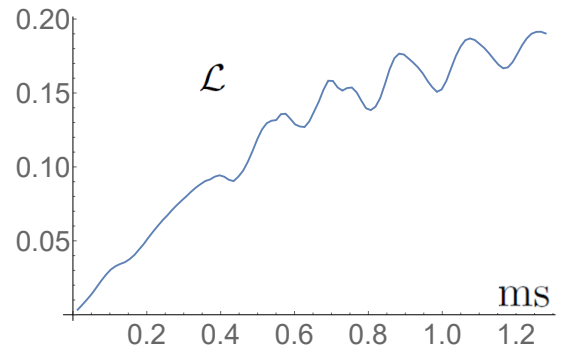

(a)

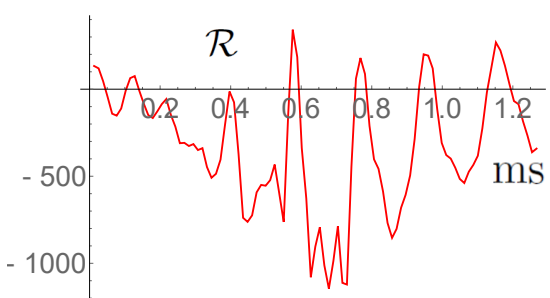

(b)

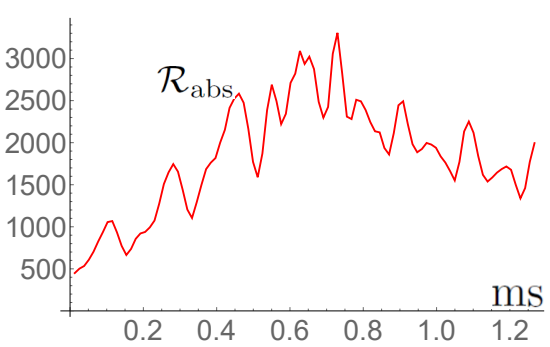

(c)

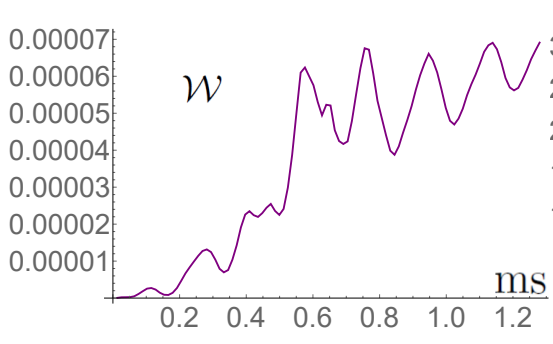

(d)

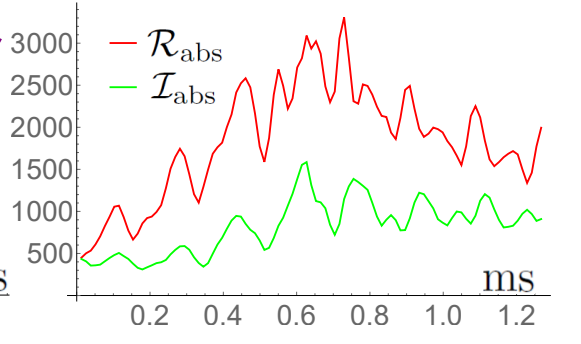

(e)

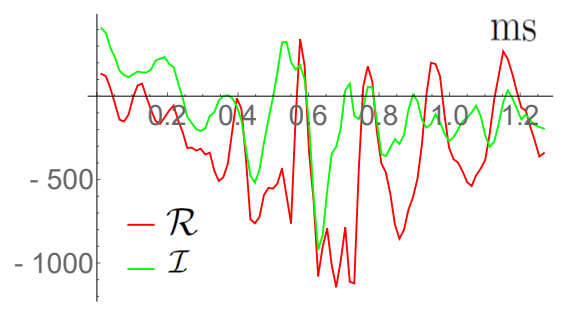

(f)

FIG. 6: Various global topological quantities charted in time. (a) $\mathcal{L}(t)$ is always positive and transitions to periodic behaviour from uniform growth. (b) $\mathcal{R}(t)$ has some oscillatory behaviour, but the mean value is consistently negative. (c) $\mathcal{R}(t)$ peaks in the middle of the field's evolution. (d) $\mathcal{W}(t)$ shows a significant rise between $t=0.5 \mathrm{~ms}$ and $t=0.64 \mathrm{~ms}$, then sets into an oscillatory cycle. (e) $\mathcal{R}_{\text {abs }}(t)$ and $\mathcal{I}_{\text {abs }}(t)$, the reconnective component is always significantly larger. (f) $\mathcal{R}(t)$ and $\mathcal{I}(t)$, generally both oscillate but during the first 400 steps the ideal contribution is net positive.

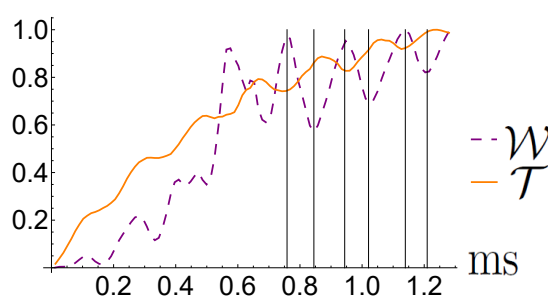

(a)

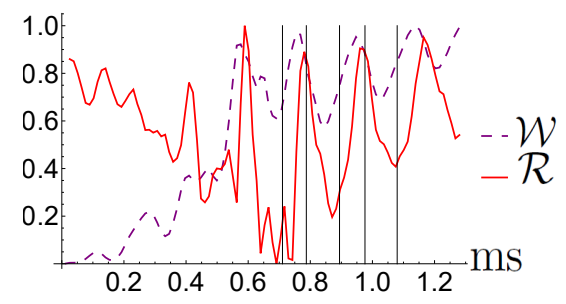

(b)

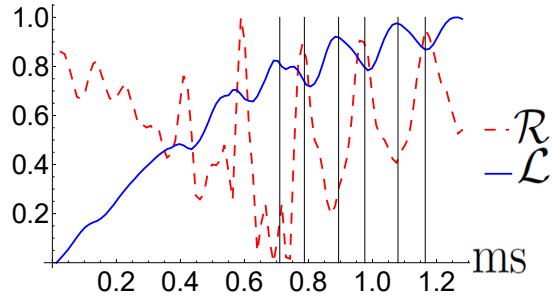

(c)

FIG. 7: Scaled plots indicating the temporal relationship between various quantities associated with B. (a) $\mathcal{T}(t)$ and $\mathcal{W}(t)$, local minima/maxima are temporally coincident (opposing). (b) $\mathcal{W}(t)$ and $\mathcal{R}(t)$, the oscillation period of the two quantities is roughly the same and local extrema in $\mathcal{W}$ always occur prior to those of $\mathcal{R}$. (c) $\mathcal{L}(t)$ and $\mathcal{R}(t)$, peaks in $\mathcal{R}(t)$ generally occur first.

There is some indication that the curves in this high $\mathcal{L}$ and $\mathcal{W}$ region are non-uniformly twisted.

To give the reader an idea of what this means for the actual three-dimensional structure, we have plotted a subset of field lines at $t=0.7936 \mathrm{~ms}$ in Figure 9. This is when the $\mathcal{L}\left(\mathbf{x}_{0}, t\right)$ structure has a spiral morphology. The field line startpoints chosen are those of high $\mathcal{W}>0.0002$, which lie on the high- $\mathcal{L}$ spiral (black), and those of medium $\mathcal{W} \in(0.0001-0.0002)$ which lie "inside" the spiral (green). The high- $\mathcal{W}$ field lines are seen to form a thin folded strip (Figure 9b), while the medium- $\mathcal{W}$ curves (Figure 9c) have a more uniformly twisted structure with less writhe. In Figure 9(d) we see that there is a reasonable degree of mixing of these two field line types.

The corresponding field lines for $t=0.896 \mathrm{~ms}$, when the $\mathcal{L}\left(\mathbf{x}_{0}, t\right)$ structure has a coherent strong central island, are shown in Figures 10(b)-(d) for the startpoints shown in Figure 10(a). The high- $\mathcal{W}$ structure still appears as a folded strip and the medium- $\mathcal{W}$ lines still have a weakly twisted structure. We note that highand medium- $\mathcal{W}$ field lines appear to be better separated into distinct regions than at $t=0.7396 \mathrm{~ms}$ (c.f. Figure $9(\mathrm{~d})$ and $10(\mathrm{~d})$ ). To quantify this apparent variation in "mixing" of high- and medium- $\mathcal{W}$ field lines, Figure 11 shows a mean "mixing ratio" between the two types of 


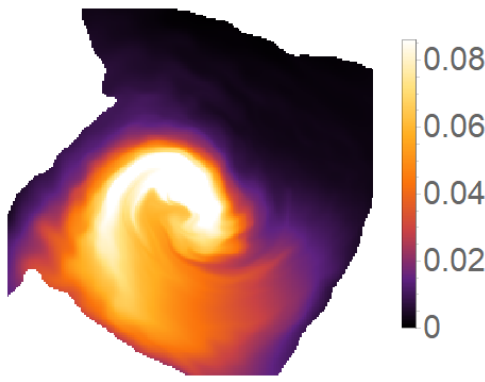

(a)

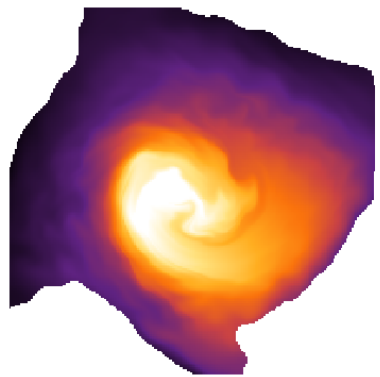

(d)

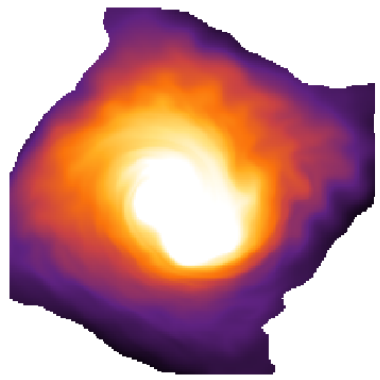

(g)

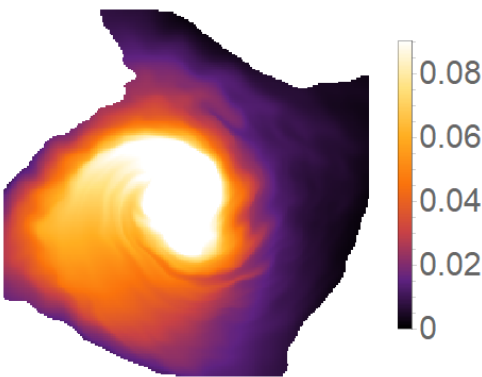

(j)

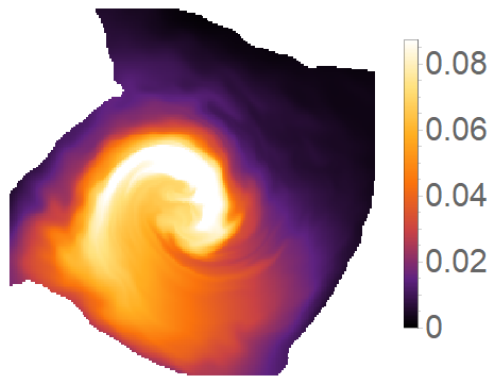

$(\mathrm{m})$

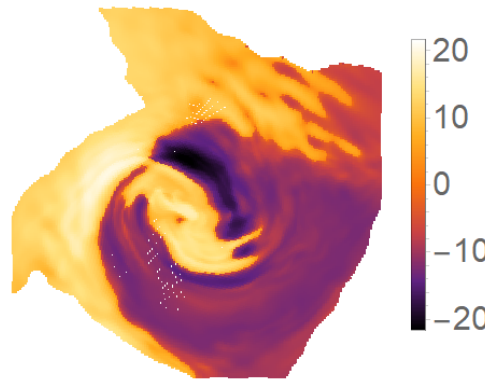

(b)
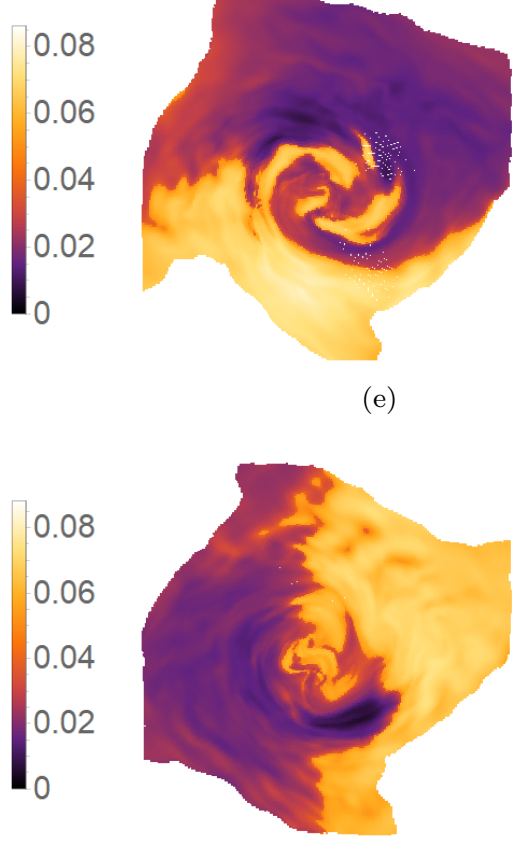

(h)

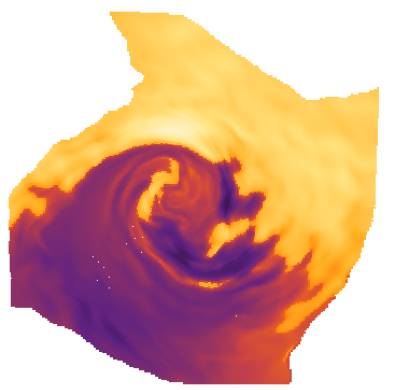

(k)

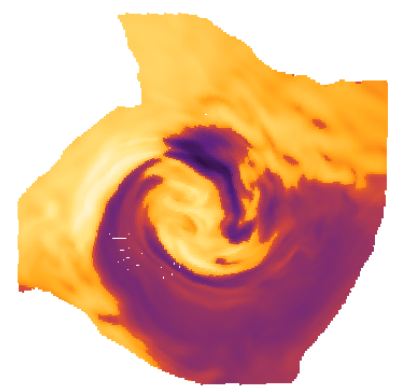

(n)

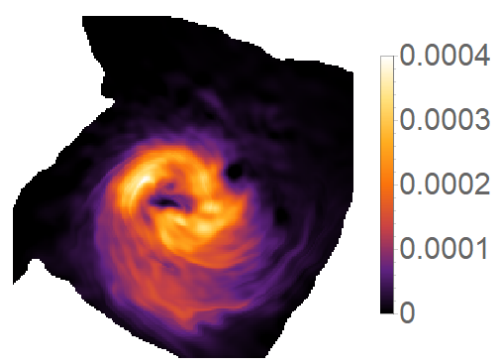

(c)
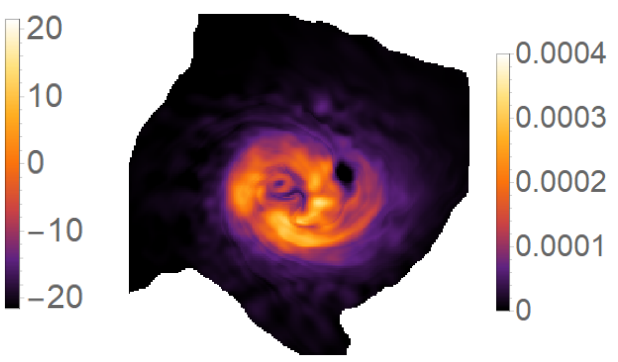

(f)
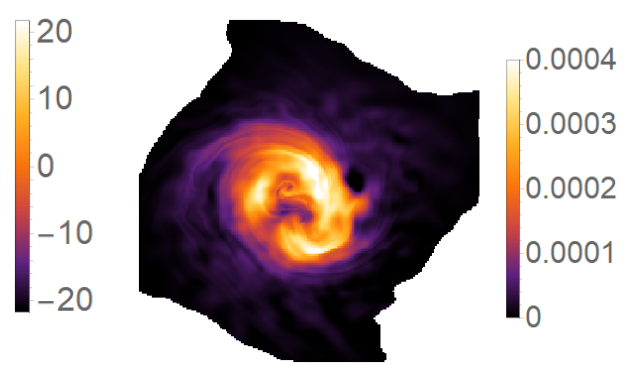

(i)
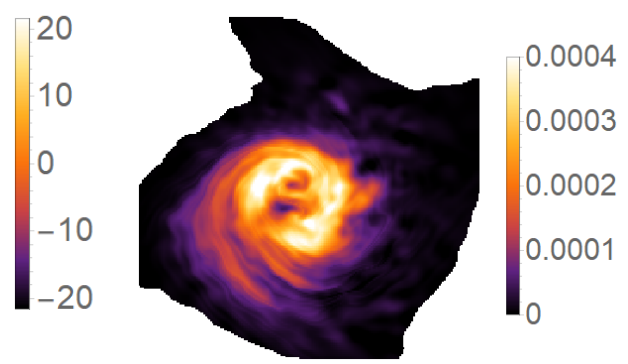

(l)
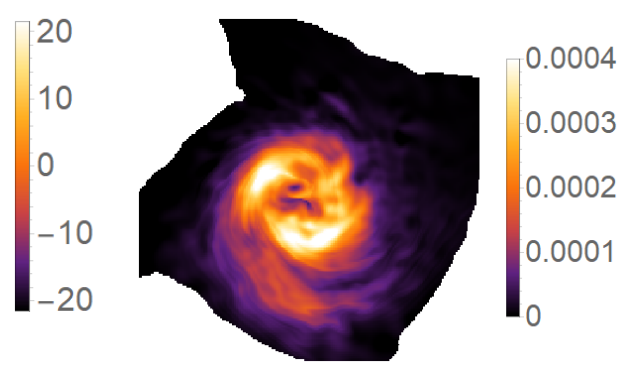

(o)

FIG. 8: Distributions of the quantities $\mathcal{L}\left(\mathrm{x}_{0}, t\right), \mathcal{R}\left(\mathrm{x}_{0}, t\right), \mathcal{W}\left(\mathbf{x}_{0}, t\right)$. The three columns represent $\mathcal{L}, \mathcal{R}$ and $\mathcal{W}$ respectively. The vertical direction follows the snapshot order

$t=0.7936 \mathrm{~ms}, 0.8448 \mathrm{~ms}, 0.896 \mathrm{~ms}, 0.9344 \mathrm{~ms}, 0.9728 \mathrm{~ms}$. The $\mathcal{L}$ distributions depict $\mathcal{L}\left(\mathrm{x}_{0}, t\right)^{2}$ and the $\mathcal{R}$ figures depict $\pm \sqrt{\left|\mathcal{R}\left(\mathbf{x}_{0}, t\right)\right|}$ so as to emphasize the features of the distributions. 


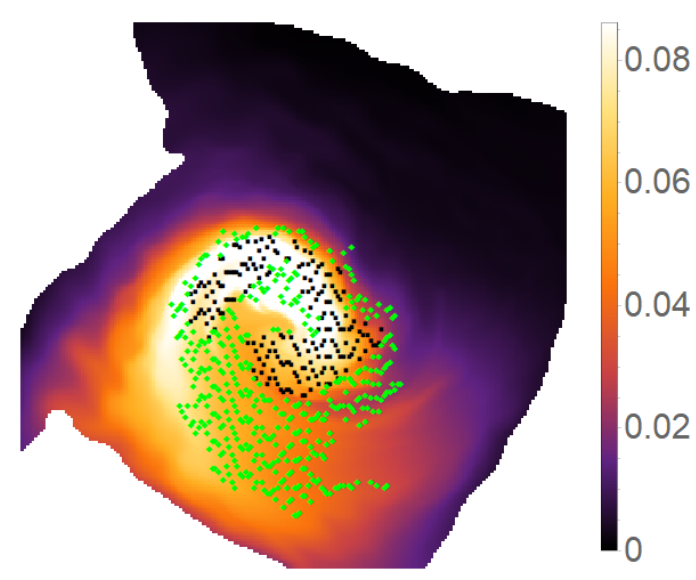

(a)

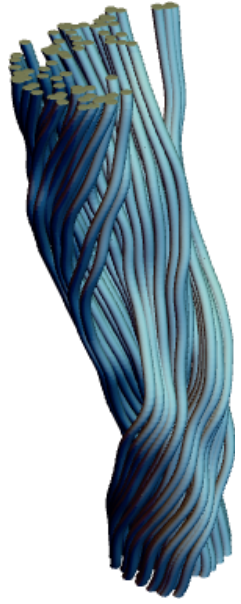

(b)

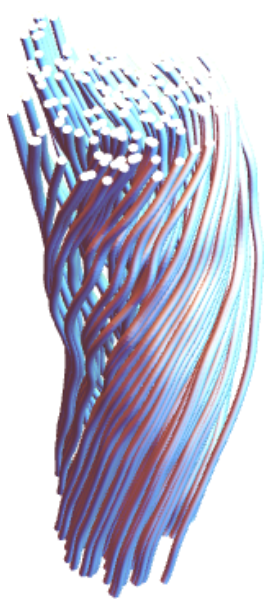

(c)

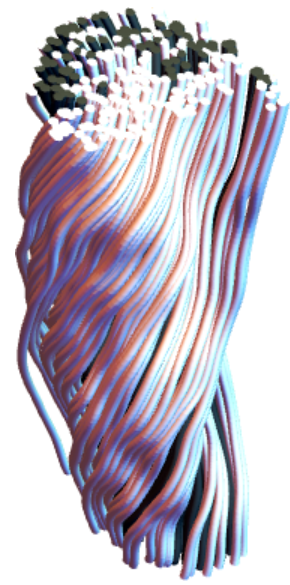

(d)
FIG. 9: Internal geometry of the central "flux rope" at $t=0.7936 \mathrm{~ms}$. (a) The set of field line startpoints, for black points $\mathcal{W}>0.0002$ and green

$0.0001<\mathcal{W}<0.0002$. (b) and (c) show these fieldline sets separately (the green fieldlines are shown as light green), (d) together.

field lines, computed at each $z$ position within the domain. Indeed, this is consistently higher for the snapshot in Figure 9 than that in Figure 10. This periodic variation in the coherence of mixing in $\mathcal{W}$ is further evidence of periodic reconnective activity in the field's core.

\section{G. Summary}

Applying the new reconnective activity measure $\mathcal{R}$, together with a set of more established geometrical quantities, has led to the following conclusions:

1. The initial magnetic field evolution establishes a bundle of high helicity $(\mathcal{L})$ field lines at the domain's center. This structure is, however, never very uniformly twisted and has complex substruc-

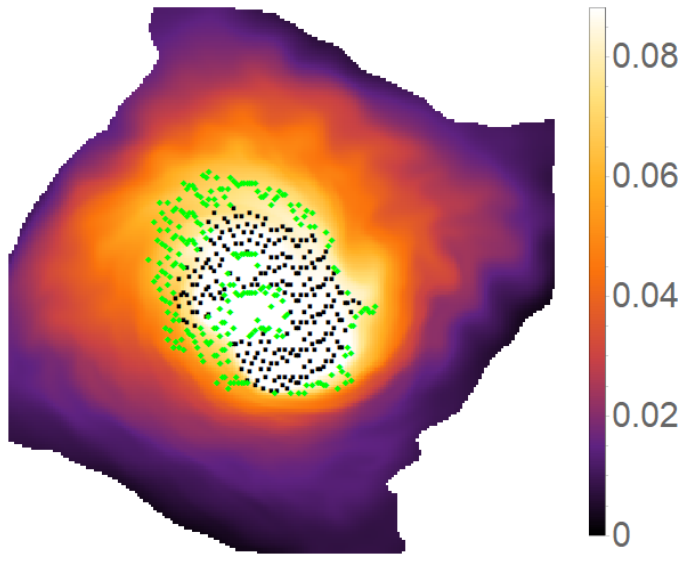

(a)

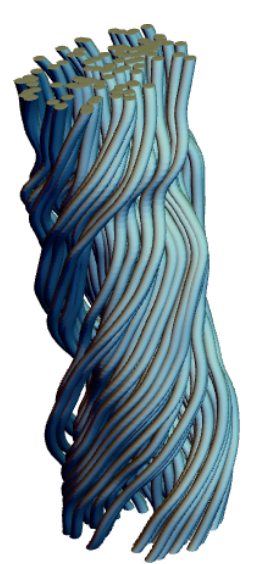

(b)

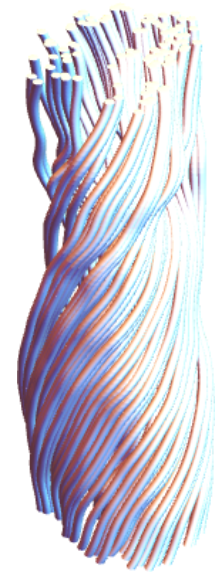

(c)

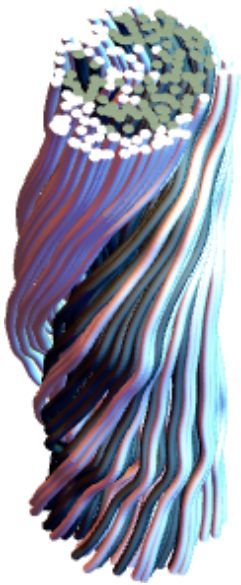

(d)
FIG. 10: Internal geometry of the central "flux rope" at $t=0.896 \mathrm{~ms}$. (a) The set of field line startpoints, for blue points $\mathcal{W}>0.0002$ and green

$0.0001<\mathcal{W}<0.0002$. (b) and (c) show these fieldline sets separately, (d) together.

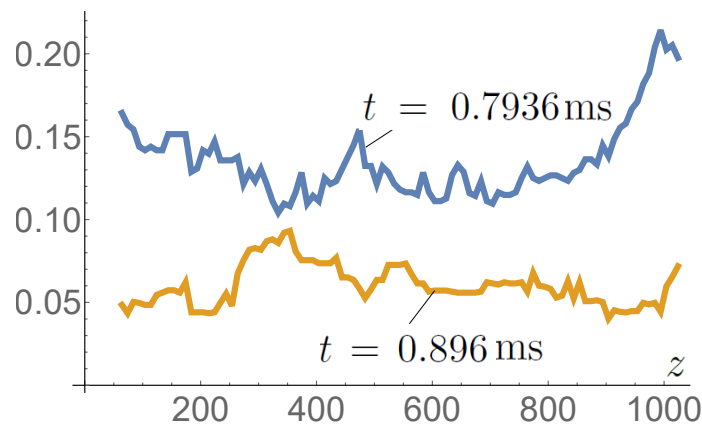

FIG. 11: Average mixing ratio of the the high and low $\mathcal{W}$ fieldlines as a function of $z$ for the field line sets shown in $9(\mathrm{~d})$ and $10(\mathrm{~d})$. This is essentially the ratio of the number of low- $\mathcal{W}$ points to the number of high- $\mathcal{W}$ points in a local neighbourhood of each high- $\mathcal{W}$ point, averaged over all such points at a given $z$. 
ture with a mixture of writhed and twisted flux elements.

2. The magnetic field then settles to steady periodic activity in which its net helicity oscillates with a fixed frequency about a uniformly growing value (see Figure 6).

3. This oscillation appears to be driven by an ideal kink instability. But this in turn drives periodically evolving non-ideal (reconnective) activity, which accounts for a most of the variation in helicity. In particular, we have verified that the oscillation in helicity is not purely the result of boundary motions, but due to periodic reconnection inside the volume.

This analysis illustrates how the quantity $\mathcal{R}\left(\mathbf{x}_{0}, t\right)$ provides a valuable additional tool for probing magnetic fields of non-trivial internal topology, adding significant information to previous studies using the total magnetic helicity $[39,50]$.

The reconnection measure $\mathcal{R}$ is defined purely in terms of fieldline geometry rather than magnetic flux, so does not require measurements of the electric field in the plasma. However, in subsequent experiments at UCLA, in which the flux ropes were further apart, the electric field (both induced and electrostatic) has been measured [20], and the peak value of field-line integrated parallel electric field (corresponding to the magnetic flux reconnection rate in GMR theory) found to be of order $6 \mathrm{~V}$. To see that this is broadly consistent with our results, we take take a peak value $\mathcal{R} \approx 10^{4} \mathrm{~s}^{-1}$, with an area of $\approx 30 \mathrm{~cm}^{2}$ (from Figure $8 \mathrm{~b}$ ). The voltage is estimated as $9.9 \times 10^{7} \mathrm{Mx} \mathrm{s}^{-1} / 10^{8}=0.99 \mathrm{~V}$. The experiments in [20] had a single dominant QSL due to to the flux rope separation, whilst in the two moon experiments analyzed here the current structure is less coherent, so lower peak voltages should be expected.

\section{CONCLUSIONS}

In section II we defined a new quantity $\mathcal{R}\left(\mathbf{x}_{0}, t\right)$ to measure reconnection in braided vector fields that stretch between two boundaries. The fundamental utility of $\mathcal{R}$ is that - assuming perfect knowledge of the vector field's evolution - the field line connectivity changes if and only if the distribution of $\mathcal{R}\left(\mathbf{x}_{0}, t\right)$ over all field lines changes. Unlike the similar field-line helicity considered for magnetic braids [32, 33], the measure $\mathcal{R}$ does not depend on the field strength, nor does it require the vector field to be divergence free. In this respect, it is more general and increases the range of potential applications beyond plasma physics.

Although we have demonstrated the technique using magnetic field data obtained from plasma experiments in the UCLA Large Plasma Device, it extracts information about changing field-line connectivity in a modelfree way, and as such has the potential for significantly wider applicability. We have also shown how to remove the contribution of an underlying ideal motion, which is relevant both for plasma physics and vortex dynamics, where one is interested only in connectivity changes with respect to the underlying fluid.

\section{ACKNOWLEDGMENTS}

The authors are indebted to W. Gekelman at UCLA for sharing experimental data and for commenting on an earlier draft of the manuscript, and to the Leverhulme Trust for financial support through Research Programme Grant RP2013-K-009 on Scientific Properties of Complex Knots. ARY also thanks STFC for financial support. We thank the anonymous referees for useful suggestions which have improved the paper.

\section{Appendix A: Proof of Theorem II.1}

To prove Theorem II.1 (Section II B), we will apply a previous result obtained for braided magnetic fields by [32] and [33]. Given some divergence-free braided vector field $\mathbf{U}(\mathbf{x})$, we define the field line mapping $F_{\mathbf{U}}: \mathcal{D}_{0} \rightarrow$ $\mathcal{D}_{h}$ by tracing field lines of $\mathbf{U}$, and define the field line helicity of a field line $\gamma\left(\mathbf{x}_{0}\right)$ by

$$
\mathcal{A}_{\mathbf{U}}\left(\mathbf{x}_{0}\right)=\int_{\gamma\left(\mathbf{x}_{0}\right)} \frac{\mathbf{A} \cdot \mathbf{U}}{U_{z}} \mathrm{~d} z .
$$

Here $\mathbf{U}=\nabla \times \mathbf{A}$. We have the following result.

Theorem A.1 ([32, 33]) Let $\mathbf{U}$ and $\mathbf{U}^{\prime}$ be two divergence-free braided vector fields, and suppose that $F_{\mathbf{U}^{\prime}}^{-1} \circ F_{\mathbf{U}}$ is the identity when restricted to the boundary $\partial \mathcal{D}_{0}$. Then $F_{\mathbf{U}^{\prime}}^{-1} \circ F_{\mathbf{U}}$ is the identity everywhere on $\mathcal{D}_{0}$ if and only if $\mathcal{A}_{\mathbf{U}}\left(\mathbf{x}_{0}\right)=\mathcal{A}_{\mathbf{U}^{\prime}}\left(\mathbf{x}_{0}\right)$ for all $\mathbf{x}_{0} \in \mathcal{D}_{0}$.

To show that Theorem II.1 follows from Theorem A.1, we will construct appropriate divergence-free vector fields $\mathbf{U}, \mathbf{U}^{\prime}$ which have the same field line curves as the original $\mathbf{B}$ at times $t$ and $t-\Delta t$, respectively, but for which $\mathcal{A}_{\mathbf{U}}\left(\mathbf{x}_{0}\right)=\mathcal{L}\left(\mathbf{x}_{0}, t\right)$ and $\mathcal{A}_{\mathbf{U}^{\prime}}\left(\mathbf{x}_{0}\right)=\mathcal{L}\left(\mathbf{x}_{0}, t-\Delta t\right)$. The result will then follow. The condition that $F_{\mathbf{U}^{\prime}}^{-1} \circ F_{\mathbf{U}}$ be the identity on the boundary is always satisfied in our case, since the two vector fields are assumed to be related by a continuous time evolution with line-tied end-points on $\mathcal{D}_{0}$ and $\mathcal{D}_{h}$.

To construct $\mathbf{U}(\mathbf{x})$ (for example), we set $\mathbf{U}(\mathbf{x})=$ $U(\mathbf{x}) \hat{\mathbf{b}}$, where $\hat{\mathbf{b}}=\mathbf{B} /|\mathbf{B}|$ is a unit vector parallel to $\mathbf{B}$ (which is well-defined since $\mathbf{B} \neq 0$ ). We then fix the magnitude $U(\mathbf{x})$ such that (i) $\nabla \cdot \mathbf{U}=0$ and (ii) $U_{z}\left(x_{1}, y_{1}, 0\right)=1$ on $\mathcal{D}_{0}$, by integrating the ODE

$$
\frac{\mathrm{d} U}{\mathrm{~d} s}=-U \nabla \cdot \hat{\mathbf{b}}
$$

along each field line with arclength $s$. Then $\mathbf{U}$ has the same field lines as $\mathbf{B}$ but is divergence free and normalized 
so that $\mathbf{U} \cdot \hat{\mathbf{z}}=1$ on $\mathcal{D}_{0}$. This works whether or not the original $\mathbf{B}$ was divergence free, provided that $\mathbf{B} \neq 0$ everywhere.

Next we use the results of [34] which show that the quantity $\mathcal{A}_{\mathbf{U}}$ can always be written in terms of the winding number $\mathcal{L}\left(\mathbf{x}_{0}\right)$. In particular there is a gauge, the winding gauge $\mathbf{A}^{\mathrm{w}}$, for which

$$
\begin{aligned}
\mathbf{A}^{\mathrm{w}} & =A_{1}^{\mathrm{w}} \hat{\mathbf{x}}_{1}+A_{2}^{\mathrm{w}} \hat{\mathbf{x}}_{2}+A_{z}^{\mathrm{w}} \hat{\mathbf{z}}, \\
A_{1}^{\mathrm{w}} & =\int_{\mathcal{D}_{z}} \frac{-B_{z}(y)\left(x_{2}-y_{2}\right)}{r^{2}} \mathrm{~d} y_{1} \mathrm{~d} y_{2}, \\
A_{2}^{\mathrm{w}} & =\int_{\mathcal{D}_{z}} \frac{B_{z}(y)\left(x_{1}-y_{1}\right)}{r^{2}} \mathrm{~d} y_{1} \mathrm{~d} y_{2}, \\
A_{z}^{\mathrm{w}} & =\int_{\mathcal{D}_{z}} \frac{B_{1}\left(x_{2}-y_{2}\right)-B_{2}\left(x_{1}-y_{1}\right)}{r^{2}} \mathrm{~d} y_{1} \mathrm{~d} y_{2}, \\
r^{2} & =\left(x_{1}-y_{1}\right)^{2}+\left(x_{2}-y_{2}\right)^{2},
\end{aligned}
$$

and

$$
\frac{\mathbf{A}^{\mathrm{w}} \cdot \mathbf{U}}{U_{z}}=\frac{1}{2 \pi} \int_{\mathcal{D}_{z}} \frac{\mathrm{d} \Theta(\widetilde{\gamma}, \gamma, z)}{\mathrm{d} z} U_{z}(\mathbf{y}) \mathrm{d}^{2} y
$$

where $\gamma$ is the field line of $\mathbf{U}$ passing through $\mathbf{x}$ and $\widetilde{\gamma}$ is the field line passing through $\mathbf{y}$ [34]. Finally we must show that the field line helicity $\mathcal{A}_{\mathbf{U}}\left(\mathbf{x}_{0}\right)$ in the winding gauge $\mathbf{A}^{\mathrm{w}}$ equals $\mathcal{L}\left(\mathbf{x}_{0}\right)$. This will follow from the fact that $\left.U_{z}\right|_{\mathcal{D}_{0}}=1$. We can pull this integral back to $\mathcal{D}_{0}$ (e.g. $[32,33])$. Integrating along $\gamma$ then gives

$$
\mathcal{A}_{\mathbf{U}}\left(\mathbf{x}_{0}\right)=\frac{1}{2 \pi} \int_{\gamma} \int_{\mathcal{D}_{0}} \frac{\mathrm{d} \Theta(\widetilde{\gamma}, \gamma, z)}{\mathrm{d} z} \mathrm{~d}^{2} y_{0} \mathrm{~d} z=\mathcal{L}\left(\mathbf{x}_{0}\right)
$$

[1] P. L. Boyland, H. Aref, and M. A. Stremler, Journal of Fluid Mechanics 403, 277 (2000).

[2] D. Kleckner and W. T. Irvine, Nature physics 9, 253 (2013).

[3] P. Comte, M. Lesieur, and E. Lamballais, Physics of Fluids A: Fluid Dynamics 4, 2761 (1992).

[4] P. Bartello, O. Métais, and M. Lesieur, Journal of Fluid Mechanics 273, 1 (1994).

[5] O. Métais, C. Flores, S. Yanase, J. J. Riley, and M. Lesieur, Journal of Fluid Mechanics 293, 47 (1995).

[6] F. Grinstein, E. Gutmark, T. Parr, D. Hanson-Parr, and U. Obeysekare, Physics of Fluids 8, 1515 (1996).

[7] D. Dabiri, Journal of Fluid Mechanics 480, 217 (2003).

[8] C. P. Broedersz and F. C. MacKintosh, Reviews of Modern Physics 86, 995 (2014).

[9] N. S. Gov, Physical Review E 78, 011916 (2008).

[10] J. Koplik and H. Levine, Physical Review Letters 71, 1375 (1993).

[11] E. Parker, The Astrophysical Journal 264, 635 (1983).

[12] A. Van Ballegooijen, The Astrophysical Journal 311, 1001 (1986).

[13] J. Klimchuk, in Physics of the Solar Corona and Transition Region (Springer, 2001) pp. 53-75.

[14] H. Peter, B. V. Gudiksen, and A. Nordlund, The Astrophysical Journal Letters 617, L85 (2004).

[15] A. Wilmot-Smith, G. Hornig, and D. Pontin, The Astrophysical Journal 704, 1288 (2009).

[16] A. Wilmot-Smith, Phil. Trans. R. Soc. A 373, 20140265 (2015).

[17] J. Granot, The Astrophysical Journal Letters 816, L20 (2016).

[18] S. Tripathi and W. Gekelman, Physical review letters 105, 075005 (2010).

[19] W. Gekelman, E. Lawrence, and B. Van Compernolle, The Astrophysical Journal 753, 131 (2012).

[20] W. Gekelman, T. De Haas, W. Daughton, B. Van Compernolle, T. Intrator, and S. Vincena, Physical review letters 116, 235101 (2016).

[21] E. Gouillart, J.-L. Thiffeault, and M. D. Finn, Physical Review E 73, 036311 (2006).
[22] H. Moffatt and A. Tsinober, Annual review of fluid mechanics 24, 281 (1992).

[23] E. Parker, The Astrophysical Journal 174, 499 (1972).

[24] J. B. Taylor, Physical Review Letters 33, 1139 (1974).

[25] C. Mellor, C. Gerrard, K. Galsgaard, A. Hood, and E. Priest, Solar Physics 227, 39 (2005).

[26] I. De Moortel and K. Galsgaard, Astronomy \& Astrophysics 451, 1101 (2006).

[27] A. Wilmot-Smith, D. Pontin, and G. Hornig, Astronomy \& Astrophysics 516, A5 (2010).

[28] D. Pontin, A. Wilmot-Smith, G. Hornig, and K. Galsgaard, Astronomy \& Astrophysics 525, A57 (2011).

[29] A. Van Ballegooijen, M. Asgari-Targhi, and M. Berger, The Astrophysical Journal 787, 87 (2014).

[30] A. J. Russell, A. R. Yeates, G. Hornig, and A. L. WilmotSmith, Physics of Plasmas 22, 032106 (2015).

[31] C. Prior and A. Yeates, Astronomy \& Astrophysics 591, A16 (2016).

[32] A. R. Yeates and G. Hornig, Physics of Plasmas 20, 012102 (2013), arXiv:1208.2286 [physics.plasm-ph].

[33] A. R. Yeates and G. Hornig, in Journal of Physics Conference Series, Journal of Physics Conference Series, Vol. 544 (2014) p. 012002, arXiv:1304.8064 [physics.plasm$\mathrm{ph}]$.

[34] C. Prior and A. Yeates, The Astrophysical Journal 787, 100 (2014).

[35] M. Hesse and K. Schindler, Journal of Geophysical Research: Space Physics 93, 5559 (1988).

[36] M. Hesse and J. Birn, Advances in Space Research 13, 249 (1993).

[37] P. Wyper and D. Pontin, Physics of Plasmas 21, 082114 (2014).

[38] P. Wyper and D. Pontin, Physics of Plasmas 21, 102102 (2014).

[39] T. DeHaas and W. Gekelman, Physics of Plasmas 24, 072108 (2017).

[40] A. Wilmot-Smith and I. De Moortel, Astronomy \& Astrophysics 473, 615 (2007).

[41] D. Mackay and A. Van Ballegooijen, The Astrophysical Journal 642, 1193 (2006). 
[42] E. E. Lawrence and W. Gekelman, Physical review letters 103, 105002 (2009).

[43] B. Van Compernolle and W. Gekelman, Physics of Plasmas 19, 102102 (2012).

[44] W. Gekelman, B. Van Compernolle, T. DeHaas, and S. Vincena, Plasma Physics and Controlled Fusion 56, 064002 (2014).

[45] M. A. Berger and C. Prior, Journal of Physics A: Mathematical and General 39, 8321 (2006).

[46] D. Leneman, W. Gekelman, and J. Maggs, Review of scientific instruments 77, 015108 (2006).

[47] D. Ryutov, I. Furno, T. Intrator, S. Abbate, and
T. Madziwa-Nussinov, Physics of plasmas 13, 032105 (2006).

[48] W. Gekelman, T. DeHaas, P. Pribyl, S. Vincena, B. Van Compernolle, and R. Sydora, Physics of Plasmas 24, 070701 (2017).

[49] G. Călugăreanu, Czechoslovak Mathematical Journal 11, 588 (1961).

[50] W. Gekelman, E. Lawrence, A. Collette, S. Vincena, B. Van Compernolle, P. Pribyl, M. Berger, and J. Campbell, Physica Scripta 2010, 014032 (2010). 